\title{
Asociación entre strain y strain rate auricular izquierdo evaluado por speckle tracking y fibrilación auricular post cirugía de revascularización miocárdica.
}

Luigi Gabrielli, Samuel Córdova, Andrés Enríquez, Paul Mc Nab, Hugo Verdejo, Iván Godoy, Ramón Corbalán

División de Enfermedades Cardiovasculares.

Hospital Clínico Pontificia Universidad Católica de Chile.

\section{Resumen:}

Introducción: La fibrilación auricular (FA) es la arritmia más común post cirugía de revascularización miocárdica (CRM) y está asociada a dilatación y disfunción auricular izquierda (AI). El strain y strain rate global longitudinal AI determinado por speckle tracking constituyen herramientas novedosas en la evaluación de la función AI.

Objetivo: evaluar el strain y strain rate global longitudinal AI en pacientes con enfermedad coronaria con indicación de CRM y su relación con el desarrollo de FA post operatoria.

Métodos: se incluyeron pacientes consecutivos con indicación de CRM, en ritmo sinusal con fracción de eyección $\geq 50 \%$. Se registraron características clínicas y ecocardiográficas con evaluación del strain AI: onda s (LASs) y strain rate: onda a (LASRa), onda s (LASRs) por speckle tracking (pre-cirugía). Se evaluó la ocurrencia de FA en el período post operatorio (una semana) mediante monitorización electrocardiográfica continua. Se utilizó t-Student, chi-cuadrado y regresión logística múltiple.

Resultados: Se incluyeron 70 pacientes, $26 \%$ presentaron FA. LASs, LASRr y LASRa estaban significativamente disminuidos en los pacientes que desarrollaron FA post CRM, LASs ( $10 \pm 1,1$ vs $24 \pm 1,2 \%$, p < $0,001), \operatorname{LASRa}(-0,6 \pm 0,1 \mathrm{vs}-1,8 \pm 0,12, \mathrm{p}<0,001)$ LASRs $(0,6 \pm 0,007$ vs $1,2 \pm 0,008, \mathrm{p}<0,001)$. Los predictores independientes de FA fueron: LASRs OR: 6,1 IC 95\% (1,3-15,2); LASRa OR: 2,4 IC 95\% (1,1-19,6); volumen AI OR: 4,67 IC 95\% (1,5-19,2) y edad > 65 años OR: 2,31 IC 95\% (1,1-15,8).

Conclusiones: LASs, LASRs y LASRa están disminudos en pacientes que desarrollan FA post CRM y LASRs, LASRa fueron predictores independientes de ésta. 


\section{Speckle tracking derived left atrial strain and strain rate in patients with post myocardial revascularization atrial fibrillation}

Background: . Atrial fibrillation (AF) is the commonest arrhythmia post coronary artery bypass grafting (CABG); it is associated to left atrial (LA) dilatation and dysfunction. Speckle tracking derived longitudinal strain and strain rate are novel techniques to evaluate LA function.

Aim: to evaluate the relation of global longitudinal LA strain and strain rate with the development of $\mathrm{AF}$ in patients undergoing $\mathrm{CABG}$.

Methods: Consecutive patients undergoing CABG with $\mathrm{LV}$ ejection $\geq 50 \%$ and sinus rhythm were included. Clinical characteristics were tabulated and LA echocardiographic speckle tracking measurements, LASs, LASRa, LARs, were used to determine LA strain and LA strain rate. Continuous ECG monitoring for 1 week was performed to assess the occurrence of AF. Student's t, chi square and multiple logistic regression were used for statistical analysis.

\section{Introducción:}

La fibrilación auricular (FA) es la arritmia más frecuente en la población adulta, afectando el $1 \%$ de la población general y más del $5 \%$ de la población mayor a 65 años ${ }^{1}$. El desarrollo de FA se asocia a deterioro significativo de la calidad de vida, así como a un incremento en la morbimortalidad que alcanza casi al doble de la población de similar edad en ritmo sinusal ${ }^{1,2}$.

Aun cuando los mecanismos que subyacen a la génesis y perpetuación de la FA no son completamente comprendidos, la hipótesis fisiopatológica actual está basada en un modelo de reentradas funcionales múltiples en un tejido auricular eléctricamente heterogéneo ${ }^{3}$. Cambios anatómicos e histológicos en la aurícula pueden favorecer el desarrollo de FA al aumentar la anisotropía eléctrica del tejido ${ }^{3,4}$.

Un modelo particular para el estudio de la FA lo constituye la FA post cirugía de revascularización miocárdica (CRM). En estos sujetos, la inflamación, estrés oxidativo y factores mecánicos propios del procedimiento quirúrgico determinan el desarrollo de FA en individuos susceptibles. Aún cuando inicialmente se suponía que la FA del postoperatorio era un fenómeno transitorio y en última instancia benigno, estudios recientes han demostrado que al igual que otras formas de FA, la FA del postoperatorio se asocia a un riesgo de morbimortalidad elevado ${ }^{5}$. En un estudio de Villarreal et al. ${ }^{6}, 6.700$ pa-
Results. 70 patients were studied; $26 \%$ developed post-operative AF. Compared to patients remaining in sinus rhythm, patients developing post-operative $\mathrm{AF}$ had lower LASs ( $10 \pm 1,1$ vs $24 \pm 1,2 \%$, p < 0,001), LASRa $(-0,6 \pm 0,1$ vs $-1,8 \pm 0,12, \mathrm{p}<0,001)$ and LASRs $(0.6 \pm$ 0.007 vs $1.2 \pm 0.008, p<0,001)$. Independent predictors of AF were LASRs (OR: 6.1, 95\% CI 1.3-15.2); LASRa (OR: 2.4, 95\% CI 1.1-19.6); LA volume (OR: 4,67, 95\% CI 1.5-19.2) and age $>65$ years (OR: $2.31,95 \%$ CI 1.1 $-15.8)$.

Conclusion.: LASs , LASRs and LASRa are lower in patients who develop AF after CABG and LASRs and LASRa were independent predictors of post-operative AF. Thus, LA strain rate assessed by speckle tracking is useful for predicting $\mathrm{AF}$ after $\mathrm{CABG}$ in addition to classic risk factors such as age and LA volume.

Keys words: Speckle tracking echocardiography, atrial strain, atrial fibrillation

cientes fueron seguidos post CRM, los pacientes con FA postoperatoria presentaron un riesgo incrementado de muerte y accidente vascular encefálico en el corto plazo, y una mortalidad elevada en el seguimiento alejado (RR 1.5, IC 95\% 1.3-1.9). Más recientemente, Mariscalco et al., demostraron que el exceso de mortalidad en el seguimiento alejado depende principalmente de eventos embólicos fatales ${ }^{7}$. Dada la alta prevalencia de la FA, que puede afectar hasta a un 30\% de los pacientes luego de cirugía de CRM, se hace evidente la necesidad de un estudio detallado de los factores predisponentes al desarrollo de la arritmia y de los elementos que determinan un sustrato susceptible para identificar apropiadamente los pacientes en riesgo y orientar efectivamente las intervenciones profilácticas para la ocurrencia de la arritmia y sus complicaciones. Evidencia de diferentes estudios clínicos sugieren un rol de numerosos factores como inflamación, estrés oxidativo, fibrosis auricular y alteración en expresión de conexinas que llevan a un sustrato proarrítmico ${ }^{8}$. Por otro lado, una serie de elementos clínicos están asociados con un aumento del riesgo de FA post CRM como la edad avanzada y un aumento del tamaño auricular izquierdo (AI), dos de los elementos más consistentemente predictores de FA en la mayoría de las series ${ }^{9}$. Además, la disfunción AI ha sido observada en pacientes con enfermedad coronaria y ha sido propuesta como un factor de riesgo potencial para el desarrollo de FA post CRM. ${ }^{10}$ 
Asociación entre strain y strain rate auricular izquierdo evaluado por speckle tracking y fibrilación auricular post cirugía...

Luigi G, et al.

En años recientes, el estudio del strain y strain rate AI por speckle tracking bidimensional ha surgido como un método novedoso para la evaluación de la función AI ${ }^{11}$. El speckle tracking es una herramienta ecocardiográfica que permite seguir el patrón de speckles cuadro por cuadro en la imagen bidimensional permitiendo la cuantificación de la deformación del tejido ${ }^{11}$. El strain y strain rate $\mathrm{AI}$ se correlaciona en forma adecuada con parámetros ecocardiográficos de evaluación de la función auricular y ha sido demostrado que el strain rate AI alterado se asocia a una mayor recurrencia de FA paroxística ${ }^{12}$. El presente estudio tiene como objetivo evaluar el strain y strain rate AI por speckle tracking bidimensional en pacientes con enfermedad coronaria (EC) e indicación de CRM y observar su relación con el desarrollo de FA post operatoria.

\section{Métodos}

\section{Población de estudio}

Se incluyeron un total de 70 pacientes con enfermedad coronaria (EC) que fueran a CRM electiva. Los criterios de inclusión fueron: ritmo sinusal preoperatorio, fracción de eyección $\geq 50$, cirugía con circulación extracorpórea.

Criterios exclusión: FA previa, síndrome coronario agudo dentro del mes previo, enfermedad inflamatoria o infecciosa activa, neoplasia, enfermedad pulmonar obstructiva crónica, insuficiencia renal (creatinina $>1,5$ $\mathrm{mg} / \mathrm{dL}$ ). Todos los sujetos firmaron un consentimiento informado aprobado por el comité de ética de nuestra institución. Se registraron las características clínicas y demográficas de todos los sujetos y se realizó un ecocardiograma transtorácico previo a la cirugía por un operador experto y ciego.

Se realizó monitorización electrocardiográfica continua durante 5 días post operatoria para la evaluación de la ocurrencia de FA. Posterior a este período se realizaron registros electrocardiográficos según sintomatología o examen físico sugerente de FA. El seguimiento se mantuvo durante todo el período de hospitalización.

Adicionalmente, en todos los sujetos, se realizaron mediciones plasmáticas de PCR ultrasensible (PCRus) como marcador de inflamación en el período preoperatorio. Se utilizó el método nefelométrico (Dade Behring BN II Nephelometer) con un límite de detección de 0,1 $\mathrm{mg} / \mathrm{L}^{13}$.

\section{Estudio ecocardiográfico}

En cada paciente se realizó un estudio ecocardiográfico transtorácico bidimensional completo por un operador experto y ciego. Se utilizó el equipo Vivid 7 General
Electric Medical Health disponible comercialmente (transductor M4S de 2.5-5 MHz). Se obtuvieron las ventanas ecocardiográficas habituales con el paciente en decúbito lateral izquierdo. Se calculó el volumen AI a partir de las visiones apicales de cuatro y dos cámaras utilizando el método biplano de discos y la masa ventricular izquierda mediante la fórmula de Devereux ${ }^{14}$. La función diastólica fue evaluada mediante el registro de las velocidades de influjo mitral con doppler pulsado y doppler tisular a nivel del anillo mitral medial y lateral en la visión cuatro cámaras apical.

\section{Strain y strain rate auricular izquierdo}

Para el estudio del strain y strain rate AI las imágenes fueron procesadas post adquisición con el programa Echopac7 versión 2.1.2 del 2008. Los bordes endocárdicos fueron trazados manualmente usando un puntero. El strain AI fue calculado usando el registro electrocardiográfico como referencia lo que permitió el reconocimiento del peak de la onda positiva sistólica (LASs) que corresponde a la función de conducto de la AI. De igual forma, en la curva del strain rate AI se identificó el peak de la onda positiva sistólica (LASRs) al inicio del sístole ventricular izquierdo y el peak de la onda negativa durante la contracción auricular (LASRa). Figura 1. El programa divide la pared de la AI en 6 segmentos y se consideró el promedio de LASs, LASRs and LASRa para el análisis.

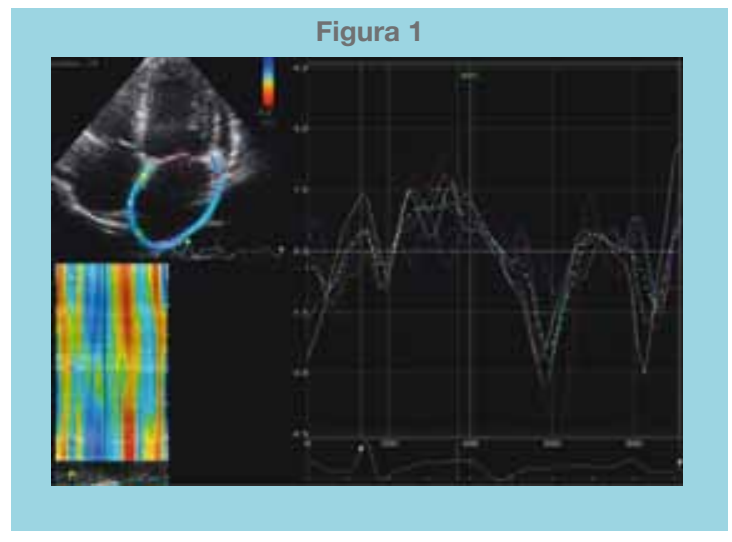

Speckle tracking bidimensional de la auricular izquierda. Curva de strain rate que muestra la onda sistólica positiva LASRs) y el peak de la onda negativa en relación a la contracción auricular (LASRa).

\section{Análisis estadístico}

Para el cálculo del tamaño muestral se utilizó el programa Power and Sample size calculations versión 
2.1.31 considerando como referencia los datos de Tsai et al 12. Con una potencia de $90 \%$, error alfa de $5 \%$ los grupos con y sin FA debían ser al menos de 15 sujetos. Las variables fueron presentadas como porcentajes y promedios \pm DS. Las diferencias entre los grupos que desarrollaron y no desarrollaron FA fueron evaluadas con chi-cuadrado y t-Student según correspondía. Se utilizó análisis por regresión logística múltiple para la evaluación de predictores independientes de ocurrencia de FA post operatoria. Un valor $\mathrm{p}<0.05$ fue considerado estadísticamente significativo.

\section{Resultados}

Un 26\% de los pacientes presentaron algún episodio de FA luego de una semana de seguimiento. Las características basales, clínicas y ecocardiográficas, se muestran en la Tabla 1. La mortalidad intrahospitalaria fue de un $0 \%$ y ningún paciente experimento complicaciones tromboembólicas. Los pacientes que desarrollaron FA postoperatoria eran significativamente mayores $(62 \pm$ 2 versus $70 \pm 2$ años; $p=0,008)$ y tenían volúmenes auriculares mayores $(30 \pm 3,8 \mathrm{ml} / \mathrm{m} 2$ versus $23 \pm 1,3$ $\mathrm{ml} / \mathrm{m} 2 ; \mathrm{p}=0,01)$ en comparación con los pacientes que no presentaron la arritmia. Además, los pacientes que presentaron FA tuvieron tiempos de circulación extracorpórea más prolongados $(103 \pm 1 \mathrm{~min}$ vs $138 \pm 1 \mathrm{~min}$; $\mathrm{p}=0,02)$. Ambos grupos eran similares con respecto al género, índice de masa corporal, prevalencia de hipertensión arterial y diabetes, niveles de PCRus e índice de masa ventricular izquierdo.

\begin{tabular}{|c|c|c|c|}
\hline & No FA $(n=52)$ & FA $(n=18)$ & $p$ \\
\hline Edad (años) & $62 \pm 2$ & $70 \pm 2$ & 0,008 \\
\hline Hipertensión (\%) & 46 & 50 & NS \\
\hline Diabetes Mellitus (\%) & 21 & 27 & NS \\
\hline IMC (kg/m2) & $26 \pm 2$ & $25 \pm 2$ & NS \\
\hline Índice masa VI (g/m2) & $120 \pm 5$ & $128 \pm 8$ & NS \\
\hline$E / A$ & $1,0 \pm 0,1$ & $1,4 \pm 0,1$ & NS \\
\hline Volumen Al (mL/m2) & $23 \pm 1,3$ & $30 \pm 3,8$ & 0,01 \\
\hline$E / e^{\prime}$ & $11 \pm 1$ & $15 \pm 2$ & 0,05 \\
\hline PCR us (mg/dL) & $5,7 \pm 1,2$ & $6,8 \pm 1,4$ & NS \\
\hline TCE (min) & $103 \pm 1$ & $138 \pm 2$ & 0,02 \\
\hline
\end{tabular}

Abreviaciones. IMC: índice de masa corporal; VI: ventrículo izquierdo; TCE: tiempo de circulación extracorpórea.

Respecto de la función AI, los pacientes que presentaron FA postoperatoria tenían menor LASs $(10 \pm 1,1$ vs $24 \pm 1,2 \%, \mathrm{p}<0,001)$, menor LASRa $(-0,6 \pm 0,1$ vs $-1,8 \pm 0,12, \mathrm{p}<0,001)$ y menor LASRs $(0,6 \pm 0,07$ vs $1,2 \pm 0,08, p<0,001)$ respecto de los pacientes que no presentaron la arritmia (Figuras 2, 3 y 4). La diferencias en las ondas del strain y strain rate se mantuvieron significativas tanto en pacientes con volumen $\mathrm{AI}<32$ $\mathrm{ml} / \mathrm{m} 2$ y volumen $\mathrm{AI}>32 \mathrm{ml} / \mathrm{m} 2$. En el análisis multivariado (Figura 5) los predictores independientes de FA postoperatoria fueron: LASRs (odd ratio 6,1, IC 95\% 1,3-15,2), LASRa (odd ratio 2.4 , IC $95 \%$ 1,1-19,6), volumen AI (odd ratio 4,67, IC 95\% 1,5-19,2) y edad $>65$ años (odd ratio 2,31, IC 95\% 1,1-15,8).

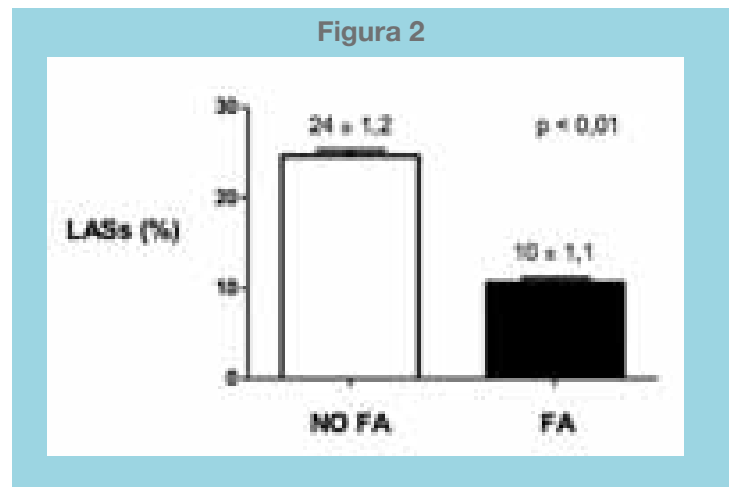

LASs en pacientes que desarrollaron FA versus los que no presentaron FA post CRM.

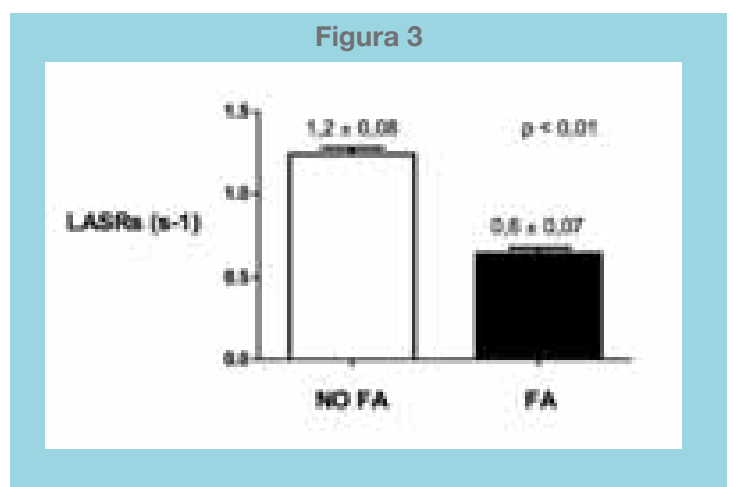

LASRs en pacientes que desarrollaron FA versus los que no presentaron FA post CRM.

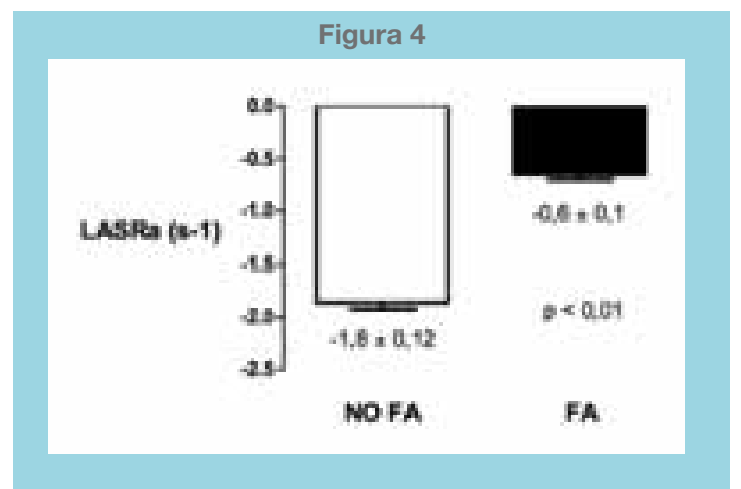

LASRa en pacientes que desarrollaron FA versus los que no presentaron FA post CRM. 


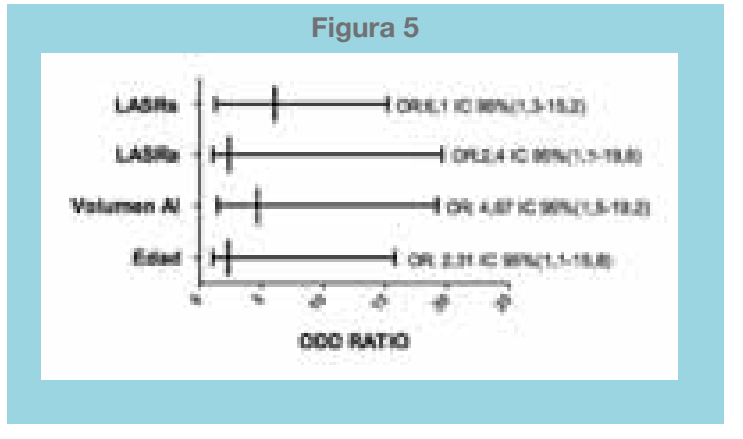

Predictores independientes de FA post cirugía de revascularización miocárdica.

\section{Discusión}

Este estudio muestra que los pacientes que presentan FA post CRM presentan parámetros de evaluación de función AI disminuidos, como son LASs, LASRs y LASRa, respecto de pacientes que no desarrollan la arritmia y esta diferencia se mantiene a distintos volúmenes auriculares, el cual junto con la edad han sido factores de riesgo clásicos para el desarrollo de FA post CRM. Además, muestra que la evaluación preoperatoria del strain rate bidimensional por speckle tracking es útil en predecir la ocurrencia de FA post CRM. Por otro lado, se muestra que los pacientes que desarrollaron FA tenían presiones de llenado del ventrículo izquierdo mayores respecto de los pacientes que no presentaron FA, al comparar la relación E/e' de ambos grupos, elemento que se asocia a crecimiento AI y que se corrobora en otros estudios ${ }^{15}$.

La presencia de disfunción AI en pacientes con EC y su asociación con el desarrollo de FA post CRM fue previamente descrito utilizando doppler tisular ${ }^{10}$. . Benedetto et al., 15 demostraron que pacientes con FA post operatoria tenían menores velocidades en el anillo mitral durante el sístole auricular en un estudio utilizando doppler tisular, un marcador que refleja la contractilidad AI. Nuestros hallazgos están en concordancia con esta evidencia anteriormente publicada. Sin embargo, es reconocido que el speckle tracking bidimensional tiene importantes ventajas sobre el estudio de tejidos con doppler tisular, incluidos la independencia del ángulo de evaluación y la tracción del tejido vecino12. El estudio de las velocidades del tejido auricular con doppler tisular no permite distinguir entre el movimiento intrínseco miocárdico y el desplazamiento pasivo debido a la tracción del tejido vecino ${ }^{16}$. Además, el estudio mediante doppler tisular limita el análisis a segmentos específicos de la pared de la AI, en cambio el speckle tracking permite el análisis de toda la pared AI otorgando una mayor información sobre la función $\mathrm{AI}^{11}$. Al contrario de nuestro estudio con speckle tracking, otros índices de evaluación de la función AI como el índice auricular (doppler) y cambios del área AI por planimetría han fallado en predecir un riesgo aumentado de FA post CRM ${ }^{17,18}$.

Las causas de la disfunción AI en la EC no están completamente esclarecidas ${ }^{19}$. Es posible que mecanismos de isquemia sobre miocitos atriales en pacientes con enfermedad ateroesclerótica de la arteria circunfleja jueguen un rol importante ${ }^{19}$, pero también es posible que anomalías estructurales como fibrosis intersticial y sobrecargas de presión auricular en presencia de disfunción sistólica y diastólica del ventrículo izquierdo tengan un rol importante ${ }^{10}$. La disfunción AI puede llevar a un mayor riesgo de FA postoperatoria a través de un remodelado eléctrico producto de un aumento en las presiones intraauriculares que resultan en una dispersión de los períodos refractarios, fibrosis y vulnerabilidad eléctrica que finalmente llevan al desarrollo de FA ${ }^{20,21}$.

En nuestro estudio no encontramos diferencia en los niveles de PCRus entre ambos grupos, a pesar de que la inflamación ha sido mostrada como un elemento de inicio y perpetuación de FA ${ }^{22}$. Probablemente, esto está dado por el modelo del estudio en donde todos los sujetos, por su patología basal, presentaban niveles de PCRus elevados haciendo más difícil lograr percibir alguna diferencia entre los grupos. Sin embargo, los pacientes con tiempos de circulación extracorpórea más prolongados, condición asociada a mayor estrés inflamatorio ${ }^{19}$, presentaron más FA en el seguimiento.

Las limitaciones de este estudio son que sólo se consideró la ocurrencia de FA en el período intrahospitalario y se puede subestimar la incidencia de FA postoperatoria y una proyección importante es correlacionar estos hallazgos con la histología AI en relación a la presencia de fibrosis en el tejido.

Las implicancias clínicas de estos hallazgos están por definirse. La incorporación del estudio de la función AI por speckle tracking podría mejorar nuestra habilidad predictiva de FA post CRM, permitiendo identificar pacientes de mayor riesgo que requerirán un mayor y más estricto seguimiento, la incorporación de medidas profilácticas de FA y eventualmente de terapia anticoagulante en los pacientes de mayor riesgo. 


\section{Referencias:}

1. LLOYD-JONES DM, WANG TJ, LEIP EP, LARSON MG, LEVY D, VASAN RS, ET AL. Lifetime risk for development of atrial fibrillation: the Framingham Heart Study. Circulation 2004;110:1042-6.

2. VERDECCHIA P, REBOLDI G, GATTOBIGIO R, BENTIVOGLIO M, BORGIONI C,ANGELI F, et al. Atrial fibrillation in hypertension: predictors and outcome. Hypertension 2003;41:218-23.

3. KORANTZOPOULOS P, KOLETTIS T, SIOGAS K, GOUDEVENOS J. Atrial fibrillation and electrical remodeling: the potential role of inflammation and oxidative stress. Med Sci Monit 2003;9:RA225-RA229.

4. THIJSSEN VL,AUSMAJ, BORGERS M. Structural remodelling during chronic atrial fibrillation: act of programmed cell survival. Cardiovasc Res 2001;52:14-24.

5. LEVY D, KANNEL WB. Postoperative atrial fibrillation and mortality: do the risks merit changes in clinical practice? J Am Coll Cardiol 2004;43:749-51.

6. VILLAREAL RP, HARIHARAN R, LIU BC, KAR B, LEE VV, ELAYDAM, et al. Postoperative atrial fibrillation and mortality after coronary artery bypass surgery. J Am Coll Cardiol 2004;43:742-8.

7. MARISCALCO G, KLERSY C, ZANOBINI M, BANACH M, FERRARESE S, BORSANI P, et al. Atrial fibrillation after isolated coronary surgery affects late survival. Circulation 2008 October 14;118:1612-8.

8. VERDEJOH, ROLDAN J, GARCIAL, DEL CA, BECERRAE, CHIONG M, ET AL. Systemic vascular cell adhesion molecule-1 predicts the occurrence of post-operative atrial fibrillation. Int J Cardiol 2010.

9. HAGHJOO M, BASIRI H, SALEK M, SADR-AMELI MA, KARGAR F, RAISSI K. Predictors of postoperative atrial fibrillation after coronary artery bypass graft surgery. Indian Pacing Electrophysiol J. 2008;8:94-101.

10. YU CM, FUNG JM, ZHANG O. Tissue Doppler echocardiographic evidence of atrial mechanical dysfunction in coronary artery disease. Int J Cardiol 2005; 105: 178-85

11. SARAIVA RM, DEMIRKOL $S$, BUAKHAMSRI A, GREENBERG N, POPOVIC ZB, THOMAS JD, et al. Left atrial strain measured by two-dimensional speckle tracking represents a new tool to evaluate left atrial function. J Am Soc Echocardiogr 2010;2:172-80.

12. TSAI WC, LEE CH, LIN CC, LIU YW, HUANG YY, LI WT, et al. Association of left atrial strain and strain rate assessed by speckle tracking echocardiography with paroxysmal atrial fibrillation. Echocardiography 2009;26:1188-94.

13. CHUNG MK, MARTIN DO, SPRECHER D. C-reactive protein elevation in patients with atrial arrhythmias: inflammatory mechanisms and persistence of atrial fibrillation. Circulation 2001;104: 2886-91.

14. LANG RM, BIERIG M, DEVEREUX RB, FLACHSKAMPF FA, FOSTER E, PELLIKKA PA. Recommendations for chamber quantification. Eur J Echocardiogr. 2006 ;7:79-108

15. BENEDETTO U, MELINA G, ROSCITANO A, CIAVARELLA GM, TONELLI E, SINATRA R. Clinical utility of tissue Doppler imaging in prediction of atrial fibrillation after coronary artery bypass grafting. Ann Thorac Surg. 2007; 83: 83-88.

16. THOMAS L, MCKAYT,BYTHK.Abnormalities of the left atrial function after cardioversion: An atrial strain rate study. Heart 2007; 93:89-95.

17. LEUNG JM, BELLOWS WH, SCHILLER NB. Impairment of left atrial function predicts post-operative atrial fibrillation after coronary artery bypass graft Eur Heart J 2004; 25:1836-1844.

18. NAKAIT,LEERJ,SCHILLER NB. The relative importance of left atrial function versus dimension in predicting atrial fibrillation after coronary artery bypass graft surgery. Am Heart J 2002;143:181186.

19. WORKMANAJ. Mechanisms of postcardiac surgery atrial fibrillation: more pieces in a difficult puzzle. Heart Rhythm. 2009;6:1423-4

20. PELLMAN J,LYON RC, SHEIKH F. Extracellular matrix remodeling in atrial fibrosis: mechanisms and implications in atrial fibrillation. J Mol Cell Cardiol. 2010;48:461-7.

21. BURSTEIN B, NATTEL S. Atrial fibrosis: mechanisms and clinical relevance in atrial fibrillation. J Am Coll Cardiol. 2008;51:802809.

22. ACEVEDO M, CORBALÁN R, BRAUN S, PEREIRA J, NAVARRETEC, GONZALEZI. C-reactive protein and atrial fibrillation: "Evidence for the presence of inflammation in the perpetuation of the arrhythmia".Int J Cardiol. 2006;108:326-331 . 